\title{
Mimicry and play initiation in 18-month-old infants
}

\author{
Christine Fawcett ${ }^{\mathrm{a}, \mathrm{b}, *}$, Ulf Liszkowski ${ }^{\mathrm{a}, \mathrm{c}}$ \\ a Max Planck Research Group Communication before Language, Max Planck Institute for Psycholinguistics, Nijmegen, The Netherlands \\ b Department of Psychology, Uppsala University, Box 1225, 75142 Uppsala, Sweden \\ c Donders Institute for Brain, Cognition and Behaviour, Radboud University, Nijmegen, The Netherlands
}

\section{A R T I C L E I N F O}

\section{Article history:}

Received 8 November 2011

Received in revised form 5 March 2012

Accepted 26 July 2012

\section{Keywords:}

Imitation

Mimicry

Play

Communication

Social cognition

\begin{abstract}
A B S T R A C T
Across two experiments, we examined the relationship between 18-month-old infants' mimicry and social behavior - particularly invitations to play with an adult play partner. In Experiment 1, we manipulated whether an adult mimicked the infant's play or not during an initial play phase. We found that infants who had been mimicked were subsequently more likely to invite the adult to join their play with a new toy. In addition, they reenacted marginally more steps from a social learning demonstration she gave. In Experiment 2 , infants had the chance to spontaneously mimic the adult during the play phase. Complementing Experiment 1, those infants who spent more time mimicking the adult were more likely to invite her to play with a new toy. This effect was specific to play and not apparent in other communicative acts, such as directing the adult's attention to an event or requesting toys. Together, the results suggest that infants use mimicry as a tool to establish social connections with others and that mimicry has specific influences on social behaviors related to initiating subsequent joint interactions.
\end{abstract}

(c) 2012 Elsevier Inc. All rights reserved.

What impact does mimicry have on infants' social interactions? Can it be used to indicate a social connection and commitment to further interaction? In adults, subtle, non-conscious mimicry, for example of body postures and gestures, has been shown to increase helping (e.g., van Baaren, Holland, Kawakami, \& van Knippenberg, 2004) and liking of another person and conversely, liking someone also leads to increased mimicry (see van Baaren, Janssen, Chartrand, \& Dijksterhuis, 2009 for a review).

Recognition of mimicry and a preference for it over other interactions is seen early in infancy. Infants distinguish mimicry from other contingent behavior by nine months of age (Agnetta \& Rochat, 2004). Already at four months of age, infants who were spontaneously mimicked by their mothers were more attentive, smiled more, and engaged in more social bidding during a later phase in which their mother displayed a still face (Bigelow \& Walden, 2009). Further, even non-human primates show recognition of mimicry (Haun \& Call, 2008). Thus, mimicry appears to be a basic feature of social interaction and could be used as a social tool from early in life.

Previous research on children's natural play shows that mimicry plays a role in social interaction with peers from early in life. Infants' synchronous imitation - or mimicry - with peers increases between the ages of 16 and 28 months (Eckerman, Davis, \& Didow, 1989) but decreases toward 5 years of age as children become more proficient in verbal interaction (Grusec \& Abramovitch, 1982; Lubin \& Field, 1981), suggesting that mimicry may be used as a form of pre-linguistic communication (see also Asendorpf \& Baudonnière, 1993). Being mimicked prompts continuation of play interactions (Grusec \& Abramovitch, 1982; Lubin \& Field, 1981) and further imitation between peers (Eckerman, 1993). Moreover, providing duplicate toys to children during natural play not only facilitates mimicry, but also results in more social interaction overall (Nadel, 2002).

\footnotetext{
* Corresponding author at: Department of Psychology, Uppsala University, Box 1225, 75142 Uppsala, Sweden. Tel.: +46 0184716337

E-mail addresses: Christine.Fawcett@psyk.uu.se (C. Fawcett), Ulf.Liszkowski@mpi.nl (U. Liszkowski).
} 
Several studies using a more experimental approach to manipulate mimicry have also shown its impacts on social interaction. For example, 14-month-olds looked and smiled more at an adult who was mimicking their actions (Meltzoff, 2007). Twoyear-olds whose actions on objects were mimicked by an adult were more likely to sustain their play with the current object, more likely to continue the same action, and more likely to create extended bouts of play (Eckerman \& Stein, 1990). Thus within an interaction, being mimicked has effects on infants' social behavior.

Another question, however, is whether mimicry has an effect beyond the immediate play situation, for example signaling a willingness or commitment to future interaction. Gräfenhain and colleagues showed that 3-but not 2-year-olds recognize verbal commitments to play together, such that they are more likely to try to reengage their partner following a verbal initiation of joint play (e.g., "let's play together") than play without such a verbal initiation (Gräfenhain, Behne, Carpenter, \& Tomasello, 2009). In contrast, for 2-year-olds in their study, simply playing next to a partner was enough to motivate later reengagement. That is, 2-year-olds were committed to play whether it was stated verbally or not. It is possible that there are nonverbal cues that younger children use to infer commitment to play. Mimicry could be a particularly salient cue serving this function of creating a social commitment to do things together in the future.

Across two experiments, we explored how mimicry during play is related to other aspects of 18-month-olds' social behavior with their adult play partner. In particular, we expected that mimicry would be related to infants' motivation to engage a partner in future play episodes, and that infants' behavior would show effects of mimicry both when they were being mimicked (Experiment 1) and when they were mimicking their partner (Experiment 2). In Experiment 1, we examined whether being mimicked leads infants to subsequently invite their partner to join them in play. In addition, we examined if infants would engage in more social learning, indicating increased willingness to learn from their play partner. In Experiment 2 , we tested whether infants' own spontaneous mimicry of their partner was related to subsequent initiations of play. In addition, we tested whether infants' mimicry during play would affect other communicative acts, such as directing attention to events and communicating a desire for a toy. Together, the two experiments examined social-interactional consequences of both being mimicked (Experiment 1 ) and mimicking another (Experiment 2) to investigate whether mimicry is used as a non-verbal tool for conveying willingness and commitment to future interaction.

\section{Experiment 1}

In Experiment 1, we examined how being mimicked by their play partner affects infants' initiation of play with that partner and their social learning from her. If infants interpret the mimicry as a signal of liking and willingness to play in the future, then they should be more likely to try to initiate play with the mimicking partner and they may also attend more closely to and learn more from their play partner's actions, showing more social learning.

\subsection{Method}

\subsubsection{Participants}

Thirty-two 18-19-month-olds participated in the study (15 girls; mean age = 18 months, 25 days, standard deviation = 15 days). An additional four infants were excluded from the analyses: three for not completing both test trials and one for experimenter error. Participants were recruited from a database of parents who expressed interest in participating in research with their child. Infants were primarily white and from middle-class families, living in a medium-sized European city or surrounding towns.

\subsubsection{Materials}

Two sets of identical toys were used in the initial play phase for the Mimicry condition. These included sets of stacking rings, three small plastic animals, a small wooden book, and a small wooden car. For the Non-Mimicry condition, one set of toys from the Mimicry condition and an additional set of toys (a stuffed animal, a wooden shoe with laces, a set of plastic blocks, and two small plastic dolls) were used. For the Inviting trial, a drum with two drumsticks was used and in the Social Learning trial, a novel wooden toy with two hinged posts and two plastic tools of different colors and shapes were used.

\subsubsection{Procedure}

A 4-min play phase was followed by two test trials: Inviting and Social Learning.

1.1.3.1. Play phase. Infants were randomly assigned to one of two conditions for the Play phase: Mimicry or Non-Mimicry. Infants sat on their parent's lap at a table next to Experimenter 1 (E1; the play partner) and across from Experimenter 2 (E2). In the Mimicry condition, the infant and E1 had identical sets of toys and E1 mimicked all the infant's actions on the toys. In the Non-Mimicry condition, the infant had the same set of toys as infants in the Mimicry condition, but E1 had a different set of toys. During the Non-Mimicry condition, E1 played with her own toys and did not mimic the infant's actions. In both conditions, E1 looked and smiled at the infant occasionally and commented on the play (e.g., "oh, this is fun") or narrated her own actions (e.g., "I'm putting a block here"). After 4 min of play, E2 put away all the toys and proceeded with the test trials.

1.1.3.2. Inviting trial. To begin the Inviting trial, E2 revealed a drum with two drumsticks. She briefly demonstrated the toy by drumming three times and saying "wow, this is great." She then gave the drum and drumsticks to the infant and quickly 
said "oh, I have to do something, I'll be right back" and left the table to wait concealed behind a screen for the remainder of the trial. Thus, E1 was left with no toy to play with and simply sat at the table gazing downward and occasionally toward the infant. If infants attempted to give her the toy, she nodded and said "oh, nice" but did not take the toy. Parents were instructed to do the same if their infant offered them the toy. After $90 \mathrm{~s}$, the trial ended and E2 returned with the materials for the next test trial and put away the drum.

1.1.3.3. Social learning trial. E2 placed the novel wooden toy on the table with the two plastic tools of different shapes and colors and looked at E1 asking "do you know how this works?". E2 then gave the toy to E1 and E1 demonstrated four target actions. First, she looked at both tools and purposefully chose one of them (counterbalanced for shape/color and side across infants). Second, she tapped the tool twice in the middle of base of the toy. Third, she pushed over each hinged post using the tool. Finally, she placed the tool back on the table and lifted the posts back into place simultaneously using her hands. She demonstrated the complete sequence of actions three times before E2 gave the toy and tools to the infant. The infant was free to act on the toy for $60 \mathrm{~s}$ before E2 put it away, ending the trial.

\subsubsection{Coding}

For the Inviting trial, infants' behavior was coded for their attempts to include E1 or their parent in play with the toy. These invitations needed to include at a minimum that the infant (1) moved the toy toward the adult, (2) gazed at her, and (3) that the coder subjectively judged that the behavior communicated a desire for E1 to engage in play together. Thus invitations often also included a vocalization or smile in addition to moving the toy and gazing at E1. If the three criteria were met again later in the trial, additional invitations were coded. For the Social Learning trial, infants' behavior was coded for the number of steps that they performed on the object, specifically: (1) choosing the same tool as E1, (2) tapping the tool on the base of the toy, (3) knocking the posts over with the tool, and (4) replacing the posts with their hand(s).

A random $25 \%$ of participants from each condition were coded by a second coder to examine reliability. Both coders were blind to the condition that the infant was in. Interclass correlations indicated that the coders' agreement on infants' behaviors was excellent (Invitations to $\mathrm{E} 1$ : $I C C=.98, p<.001$; Invitations to parent: $I C C=1.00, p<.001$; Social learning steps: ICC $=1.00, p<.001)$.

The Play phases of another random 25\% of participants were coded to ensure that E1's behavior during the play phase was equally social toward the infant regardless of whether she was imitating or not. There were no significant differences in her frequency of speech, smiles, or looks to the infant across conditions (all ps >.10).

\subsection{Results and discussion}

To examine our hypothesis that infants who were mimicked in the play phase would be more likely to invite their partner to play later, we used a linear regression model with a Poisson distribution. The Poisson distribution was used since it is appropriate for count data that are not normally distributed. The model was fit using the GLM function of the R package stats (R Development Core Team, 2009). The initial model of invitations to E1 showed that infants invited more often in the Mimicry $(M=0.75)$ than the Non-Mimicry $(M=0.19)$ condition $(z=-2.15, p=.03$, see Fig. 1$)$. As a control, a second model examining invitations to infants' parents showed that they did not differ significantly by condition (Mimicry: $M=.93$; NonMimicry: $M=.75 ; z=-0.58, p=.57$ ). While the rate of spontaneous inviting is somewhat low (approximately half of infants in either condition invited at all), the significant difference in mean number of invitations suggests that of the infants who did invite, those in the Mimicry condition were more persistent and motivated to invite even after their offer was not accepted. Together, these results reveal that infants are more motivated to invite someone to play if that person mimicked them earlier and that the increase in inviting behavior does not generalize to other individuals.

Next we explored whether infants would also learn more from E1 if she had mimicked them earlier. We compared the number of demonstrated steps out of four that the infants repeated while playing with the toy ${ }^{1}$ and found a marginally significant effect for infants to repeat more steps in the Mimicry $(M=2.63)$ than the Non-Mimicry $(M=1.94)$ condition $(t(30)=1.92, p=.06$, see Fig. 2). This suggests that infants may show an increase in social learning from someone who mimicked them during play, though the effect is not as strong as for inviting play. It is possible that mimicry has the most direct effects on social behavior that is related to further play. If mimicry creates a social bond for play interactions, then the most significant effects should be seen for initiating further play and less strong effects should be seen for other aspects of social behavior, such as social learning.

The initial finding that being mimicked affects infants' social behavior supports the proposal that mimicry creates a social connection between play partners, signaling liking and willingness for future interaction. A further question, which we addressed in Experiment 2, is whether infants also initiate mimicry during play to establish such a social bond. If so, we should observe similar relations between infants' own spontaneous mimicry of their play partner and their later invitations of play. In addition, we investigated whether this social bond would extend to other communicative situations, such as directing attention to events or requesting toys.

\footnotetext{
${ }^{1}$ Infants repeated the four different steps approximately equally often, suggesting that they were similar in their ease and interestingness (Tool choice $n=21$; Tapping $n=21$; Push $n=12$; Lift $n=27 ; \chi^{2}(3)=5.67, p=.13$ ).
} 


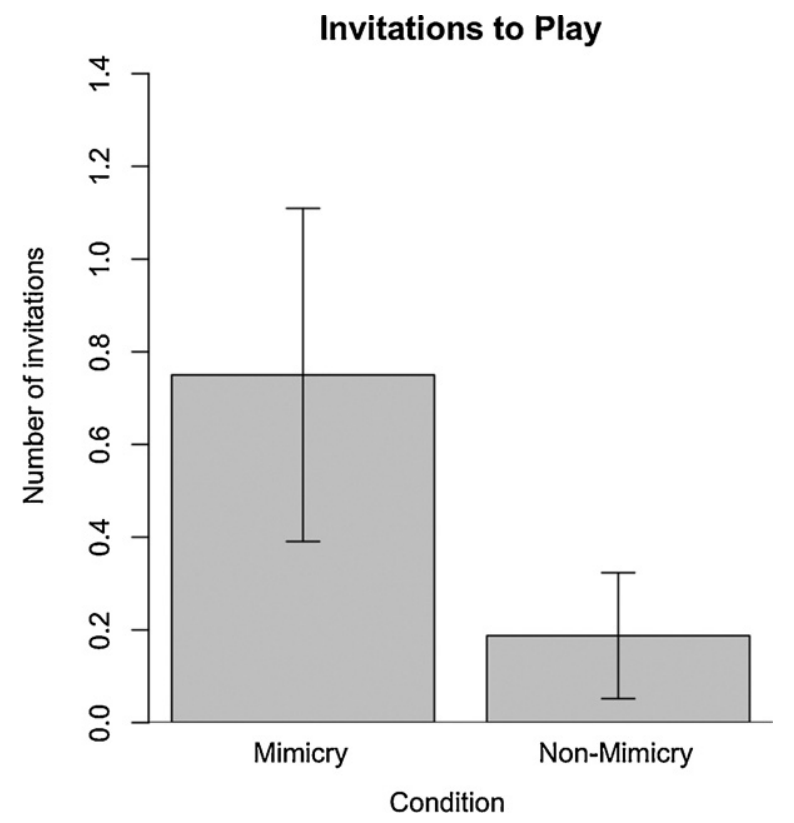

Fig. 1. Infants' rate of inviting play with a new toy to Experimenter 1 for the Mimicry and Non-Mimicry conditions.

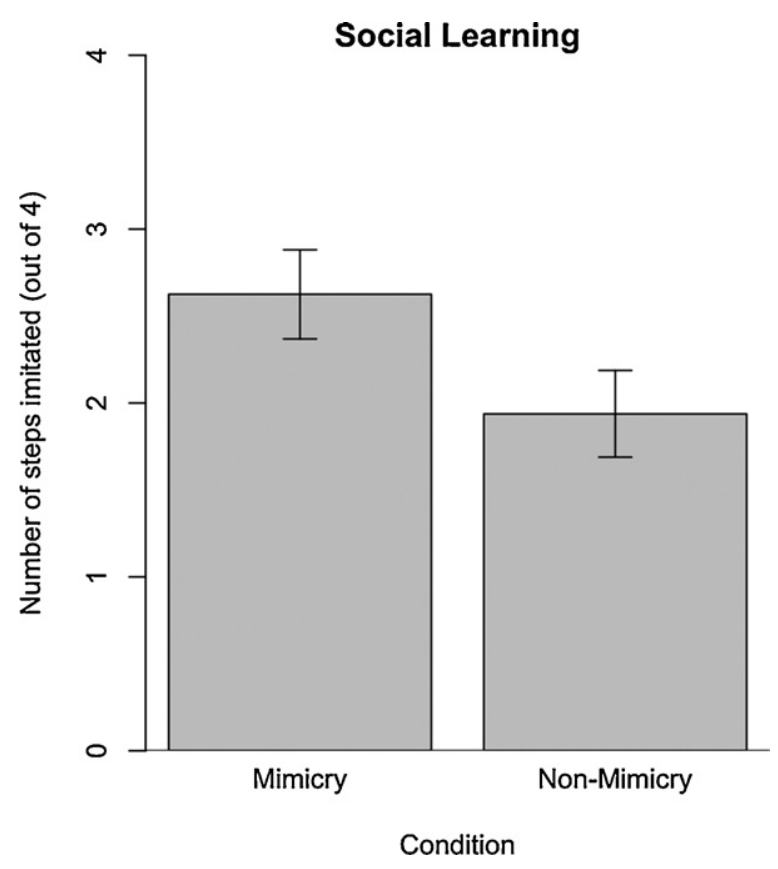

Fig. 2. Infants' social learning for the Mimicry and Non-Mimicry conditions.

\section{Experiment 2}

In Experiment 2, we examined whether infants use mimicry as a way to communicate a social connection to their partner. That is, we compared infants' spontaneous amount of mimicry of their partner's actions during a play phase to their subsequent initiation of play with her. We expected that infants who spent more time mimicking the adult during play would also subsequently initiate more play. Our additional measures of social behavior examined infants' willingness to direct their partner's attention to an interesting event (e.g., Liszkowski, Carpenter, \& Tomasello, 2007) and to request toys from her. These measures were used to examine the range of influence that mimicry has on social behavior. We chose not to use a social learning task as in Experiment 1, since the common basis of imitation of their partner could inflate the relationship between the two measures. 
Table 1

Experimenter actions on toys in Experiment 2.

\begin{tabular}{lll}
\hline Toy & Action 1 & Action 2 \\
\hline Small wooden book & Turn pages & Slide book back and forth \\
Ring stacking toy & Stack rings and take them off & Tap rings together \\
Music box & Turn handle to make music & Point to dots on the side of the music box \\
Wooden numbers & Attach and remove numbers with velcro & Scratch velcro on the board with finger \\
\hline
\end{tabular}

\subsection{Method}

\subsubsection{Participants}

A new group of thirty-two 18-19-month-olds participated in the study (15 girls; mean age = 19 months, 4 days, standard deviation = 5 days). An additional three infants were excluded from the analyses for not completing all three test trials. Participants were recruited as in Experiment 1.

\subsubsection{Materials}

For the Play phase, two wooden boards with four identical toys attached to them were used. The toys were a small wooden book with pictures of animals, a post with two rings for stacking, a music box operated by turning a handle on the top, and two wooden numbers that could be attached to the board with Velcro. We chose to have the toys attached to a board for this experiment since it kept all items within reach for the infant, making it possible for the infant to mimic E1 at any time. For the Inviting trial, the same drum and sticks were used as in Experiment 1. For the Directing trial, three large puppets were used and for the Requesting trial, four pairs of small toys were used (e.g., a stuffed rabbit and a wooden car).

\subsubsection{Procedure}

Following the 4-min Play phase, three test trials - Inviting, Directing, and Requesting - were presented in counterbalanced order.

2.1.3.1. Play phase. As in Experiment 1, infants sat on their parent's lap at a table next to E1 (the play partner) and across from E2. To begin the Play phase, E2 gave a toy board to the infant and another to E1. E1 played in a predetermined manner. She played with each toy for $30 \mathrm{~s}$ at a time, the first $15 \mathrm{~s}$ performing one action, and the next $15 \mathrm{~s}$ performing the second action. Thus, over the entire 4-min Play phase, she acted on each toy twice. Her actions were ones that were simple enough for infants to perform but were somewhat novel given the nature of the toys (see Table 1 for a complete list of actions). Infants were free to play however they chose during the play phase, though if they tried to play with items on E1's play board, E2 redirected their attention to their own board. At the end of Play phase, all toys were removed from the table.

2.1.3.2. Inviting trial. The Inviting trial proceeded as in Experiment 1, except that after $75 \mathrm{~s}$, E1 prompted the infant by saying, "oh, that's a nice toy, can I play?" and put her hand out with her palm up. If the infant did not respond, a second prompt of "can I play?" was given after $5 \mathrm{~s}$. The trial ended after a total of $90 \mathrm{~s}$. Before the prompt, E1 gazed downward and did not take the toy if it was offered (as in Experiment 1). However, after the prompt, she did take the toy from the infant if it was offered and played for a few seconds before ending the trial.

2.1.3.3. Directing trial. For the Directing trial, E2 was behind a screen at the side of the room and E1 moved to sit in E2's place at the table. E2 operated three puppets from behind the screen, raising them up one at a time so that they were visible to the infant, but not to E1 (since they were slightly behind her). Each time E2 raised a puppet, she made a sound (e.g., "Hi there!") to ensure that the infant saw the puppet. E1 continued to sit facing the infant and looking downward at the table. Regardless of the infant's behavior, after $15 \mathrm{~s}$, one puppet was lowered back behind the screen and E1 looked up at the infant and around the room, saying "hmm, what?" to indicate that she was aware that something was going on, but not what. After she looked down again, the next puppet was raised. E1's reaction served to encourage infants to continue directing her attention and not become frustrated that she was not responding to their communicative attempts. The trial continued for $90 \mathrm{~s}$.

2.1.3.4. Requesting trial. For the Requesting trial, E2 waited behind the screen and E1 sat across from the infant. E1 held out a pair of toys, one in each hand, and said "oh, these are nice, would you like one?". She looked at each of the toys and then to the infant while asking the question. After the infant made a clear request for a toy (e.g., by reaching or gesturing), she gave the toy to the infant to play for a few seconds. She retrieved that toy before revealing the next pair of toys. Four pairs were presented in total. If the infant made no request after $15 \mathrm{~s}$, that pair was put away and the next pair was revealed.

\subsubsection{Coding}

Since infants' mimicry - unlike the adult's mimicry in Experiment 1 - could not be experimentally manipulated, we coded for the amount of time infants spent acting on any toy on their toy board and the amount of time spent doing the same 


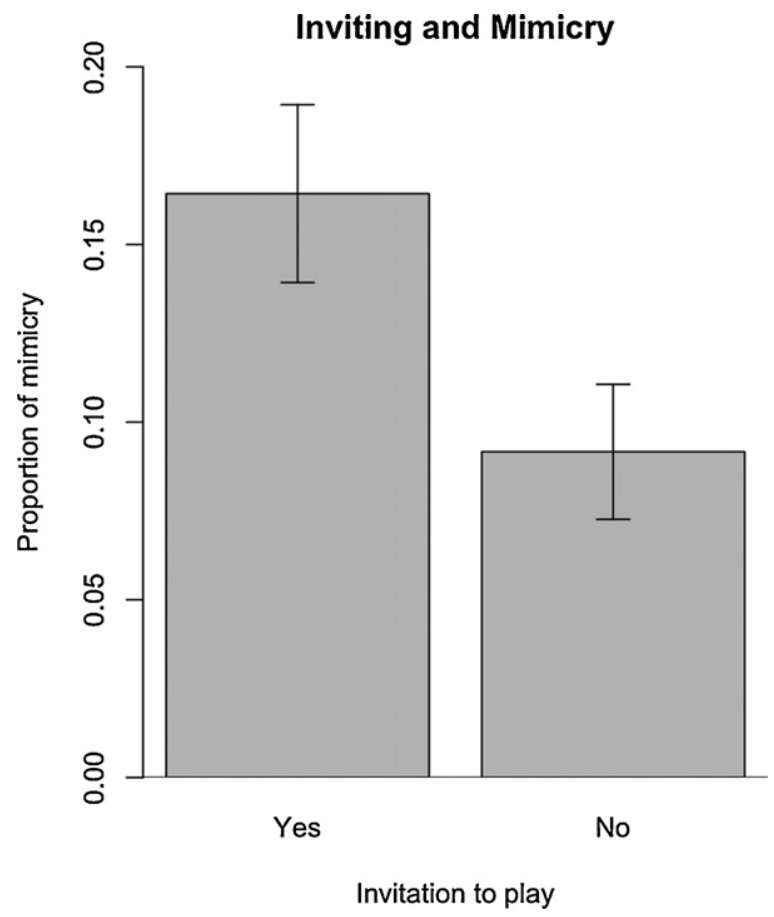

Fig. 3. Proportion of infants' spontaneous mimicry of Experimenter 1 for infants who invited her to play with a new toy and those who did not.

action with a toy as E1 (i.e., mimicking her). These durations were used to calculate the proportion of time that infants spent mimicking E1 during their play. Using infants' proportion of imitation during play seemed to be the best way to capture their motivation to mimic E1's actions over the course of their ongoing play and this measure has previously been used to study infants' peer imitation during natural play (Asendorpf \& Baudonnière, 1993).

For the Inviting trial, infants' behavior was coded for their attempts to include E1 or their parent in play with the toy as in Experiment 1. Both spontaneous invitations and invitations following E1's prompts at the end of trial were coded.

For the Directing trial, infants' behavior was coded for their attempts to direct E1 to look at the puppet that was out of her view. Similar to the coding for invitations, directing was judged subjectively by the coders, but needed to include at a minimum that the infant gazed at E1 either just before or after gazing at the puppet and either gestured toward the puppet or vocalized.

For the Requesting trial, infants' attempts to request one or both toys were coded for each toy pair. If infants made any gesture (e.g., reach or point) toward the toys, it was considered a request.

A random $25 \%$ of participants were coded by a second coder to examine reliability. Both coders first coded all of the infants' test trials before returning to each video in a random order to code the play phases. This eliminated the possibility of bias in interpreting infants' test trial behavior based on their play phase behavior. The coders' agreement on infants' behaviors was overall very good (Proportion of mimicry: ICC $=.97, p<.001$; Invitations to E1: ICC $=1.0, p<.001$; Invitations to parent: $I C C=1.0, p<.001$; Invitations to E1 following prompt: $I C C=1.0, p<.001$; Directing: $I C C=.65 p=.02$; Requests: $I C C=.63$, $p=.03)$.

\subsection{Results and discussion}

The proportion of time infants spent mimicking E1 ranged from 0 to .38 of their time acting on the objects $(M=.12, s=.09)$. Only 3 infants never mimicked E1. While not every infant performed each of E1's eight possible actions on the objects, as a group, the infants did perform all of her actions. Thus, the actions themselves were appropriate for infants of this age.

Our primary hypothesis was that infants who mimicked E1 more would be more likely to invite her to play. We analyzed infants' invitations to E1 following her prompt since only one infant spontaneously invited E1 to play. Fourteen out of 32 infants (43.8\%) invited E1 to play following her prompt, including the one infant who invited her spontaneously. Thirteen of the 14 infants responded after E1's first prompt. A nonparametric Wilcoxon summed ranks test was used to compare the amount of time spent mimicking E1 by infants who either did or did not invite her to play later. The test revealed that infants who invited E1 following her prompt had mimicked more of her actions in the previous play phase $(W=184, p=.03)$. Specifically, infants who invited E1 to join in play had mimicked her during an average of $16 \%$ of their play, compared to an average of $9 \%$ for those who did not invite her (see Fig. 3). Thus, as in Experiment 1, we found a significant relation between mimicry and inviting play. Importantly, in Experiment 2 infants spontaneously initiated the mimicry. This suggests 
that infants not only respond to being mimicked, but also actively use mimicry to establish a social connection and signal willingness for further play with a partner.

We could not compare invitations to parents as we did in Experiment 1 since parents did not prompt their infant and only 5 infants invited their parent spontaneously. As for the difference in spontaneous inviting between Experiments 1 and 2 , it is possible that the arrangement of toys in the play phase could have affected this measure. Whereas in Experiment 1 toys were loose on the table, in Experiment 2 toys were clearly separated on wooden boards for the infant and E1. This context could have reinforced that toys given to the infants were for them and decreased motivation to spontaneously invite the partner when they received a new toy later. This explanation is supported by our observation that infants' spontaneous inviting to their parents was also lower in Experiment $2(M=.19)$ than Experiment $1(M=.84)$, indicating that the difference in infants' inviting behavior was more likely an effect of the situation than their interest in their partner. In addition, there could also be an influence of having experienced much less mimicry in Experiment 2 compared to Experiment 1 (12\% vs. $100 \%)$, which in turn decreased its influence on their behavior.

Next we tested whether infants who mimicked E1 more were also more likely to direct her attention to the appearance of the puppets. Twenty-five out of the 32 infants (78.1\%) directed E1 at least once $(M=2.03, s=2.13)$. A Spearman's rank correlation test found no relation between the number of times an infant directed E1's attention to the puppets and the proportion of time they mimicked her play (Spearman's $\rho=.13, p=.49$ ). Infants' likelihood of communicating about the puppet's presence with E1 was thus not related to how much they mimicked her during play. Further, we tested whether infants who mimicked more were more likely to request toys for themselves. All infants requested at least once $(M=3.31$ out of 4 trials, $s=1.33$ ). Again, a Spearman's rank correlation found no relation between number of requests and mimicry (Spearman's $\rho=-.01, p=.95$ ), however with the high mean rate of requesting, it is also possible that there was a ceiling effect for this task. Thus, the amount that infants mimicked the play partner does not seem to be related to their likelihood of requesting items from her. Our secondary findings then reveal that mimicry is not simply indexing how likely infants are to want to interact with the play partner generally, or how comfortable they are in the experimental situation.

Experiment 2 shows that infants spontaneously mimic the play of their partner and this mimicry is related to their desire to initiate further play with that partner. We did not find relations to other social behavior, such as requesting items or directing attention. It is possible that the relation between infants' mimicry and subsequent social behaviors may be specific to acting together in play, as opposed to interacting and communicating more generally. As a whole, the findings give further support that mimicry is used early in life to signal social commitment to play together.

\section{General discussion}

Eighteen-month-old infants' initiation of play with a partner is related to previously being mimicked by her and spontaneously mimicking her themselves. We expected that creating a social connection through mimicry would be most influential on measures of desire to play together. In Experiment 1, infants were more willing to invite an adult to play with a new toy when she had mimicked their play earlier. In Experiment 2, infants who spontaneously mimicked more of the adult's play were more likely to invite her to play with a new toy later. These findings suggest that infants recognize and use mimicry as a way to communicate a social connection and commitment to future interaction.

Our secondary measures were either only marginally related to being mimicked (Experiment 1 ) or unrelated to mimicking (Experiment 2). Since the absence of findings is not indicative of independence, it is possible that with more sensitive measures, a stronger manipulation of mimicry (e.g., a longer play phase), or a more immediate test after mimicry, effects would increase. However, it is also possible that the effect of mimicry could be largely specific to other play situations, at least early in childhood, leading to a weaker relationship between mimicry and other forms of social interaction involving learning or communicating.

A very recent study shows that being mimicked during play increases also infants' willingness to help another person - either the person who mimicked them or someone else (Carpenter, Uebel, \& Tomasello, submitted for publication). That study complements our findings in that helping could be seen as similar to our play initiation tasks, in which the infant offers an item to another person in a prosocial manner. However, the helping behavior in Carpenter and colleagues' task was also different in that it involved giving back an object that had been in the other's possession, while our task required infants to offer an object that was in their own possession. This difference might help to explain the lower overall rate of our inviting behavior compared to their helping behavior.

We have proposed that a social connection created through mimicry - either when being mimicked or when choosing to mimic another - leads infants to initiate further play interactions. It is unlikely that infants' positive mood, rather than a social connection with their partner, underlies or mediates these effects. All infants in both experiments completed the play phase, suggesting that they were all engaged and enjoyed playing and did not show significant differences in mood. Further, the mimicry study of Carpenter and colleagues (submitted) also found no mood differences for infants who were mimicked, as measured by parent report. Another question is whether the effects of mimicry in infants and young children are based on the same processes as those in adults. One primary difference in the mimicry studied in adults and that studied in children is its subtleness. In adults and older children, obvious mimicry can actually be seen negatively, whereas young children seem to enjoy it. It could be that young children without language skills use mimicry as a more explicit form of intentional communication, whereas later in life, mimicry becomes a more subtle non-conscious way of communicating social alignment. 
The current research extended and elaborated on previous findings suggesting that infants respond to and use mimicry as a tool for social connection with others. Our findings show that mimicry is related to future willingness to play together, both when the infant is mimicked and when the infant mimics another. Mimicry thus seems to have a specific influence on infants' social behavior, signaling willingness to play again in the future, and could serve as a non-verbal form of committing to joint interaction.

\section{References}

Agnetta, B., \& Rochat, P. (2004). Imitative games by 9-, 14-, and 18-month-old infants. Infancy: 6., (1), 1-36.

Asendorpf, J. B., \& Baudonnière, P. M. (1993). Self-awareness and other-awareness: Mirror self-recognition and synchronic imitation among unfamiliar peers. Developmental Psychology: 29., (1), 88-95.

Bigelow, A. E., \& Walden, L. M. (2009). Infants' response to maternal mirroring in the still face and replay tasks. Infancy: $14 .$, (5), 526-549.

Carpenter, M., Uebel, J., \& Tomasello, M. Being mimicked increases prosocial behavior in 18-month-old infants, submitted for publication.

Eckerman, C. O. (1993). Imitation and toddlers' achievement of coordinated action with others. New Perspectives in Early Communicative Development, $116-138$.

Eckerman, C. O., Davis, C. C., \& Didow, S. M. (1989). Toddlers' emerging ways of achieving social coordinations with a peer. Child Development: 60., (2), $440-453$.

Eckerman, C. O., \& Stein, M. R. (1990). How imitation begets imitation and toddlers' generation of games. Developmental Psychology: 26., (3), 370-378.

Gräfenhain, M., Behne, T., Carpenter, M., \& Tomasello, M. (2009). Young children's understanding of joint commitments. Developmental Psychology: 45., (5), 1430.

Grusec, J. E., \& Abramovitch, R. (1982). Imitation of peers and adults in a natural setting: A functional analysis. Child Development: 53., (3), 636-642.

Haun, D., \& Call, J. (2008). Imitation recognition in great apes. Current Biology: 18., (7), R288-R290.

Liszkowski, U., Carpenter, M., \& Tomasello, M. (2007). Pointing out new news, old news, and absent referents at 12 months of age. Developmental Science: 10., (2), F1-F7.

Lubin, L., \& Field, T. (1981). Imitation during preschool peer interaction. International Journal of Behavioral Development: $4 .$, (4), 443.

Meltzoff, A. N. (2007). Like me: A foundation for social cognition. Developmental Science: 10., (1), 126-134. http://dx.doi.org/10.1111/desc.2007.10.issue-1

Nadel, J. (2002). Imitation and imitation recognition: Functional use in preverbal infants and nonverbal children with autism. In A. N. Meltzoff, \& W. Prinz (Eds.), The imitative mind: Development, evolution, and brain bases (pp. 42-62). Cambridge University Press.

R Development Core Team (2009). R: A language and environment for statistical computing. R Foundation for Statistical Computing, Vienna, Austria. ISBN 3-900051-07-0, URL http://www.R-project.org.

Van Baaren, R. B., Holland, R. W., Kawakami, K., \& van Knippenberg, A. (2004). Mimicry and prosocial behavior. Psychological Science: 15. ., (1), 71.

Van Baaren, R., Janssen, L., Chartrand, T. L., \& Dijksterhuis, A. (2009). Where is the love? The social aspects of mimicry. Philosophical Transactions of the Royal Society B: Biological Sciences: 364., (1528), 2381. 